PROCEEDINGS OF THE

AMERICAN MATHEMATICAL SOCIETY

Volume 137, Number 4, April 2009, Pages 1499-1507

S 0002-9939(08)09649-4

Article electronically published on October 23, 2008

\title{
ORBIT-COUNTING FOR NILPOTENT GROUP SHIFTS
}

\author{
RICHARD MILES AND THOMAS WARD
}

(Communicated by Jane M. Hawkins)

\begin{abstract}
We study the asymptotic behaviour of the orbit-counting function and a dynamical Mertens' theorem for the full $G$-shift for a finitely-generated torsion-free nilpotent group $G$. Using bounds for the Möbius function on the lattice of subgroups of finite index and known subgroup growth estimates, we find a single asymptotic of the shape
\end{abstract}

$$
\sum_{|\tau| \leqslant N} \frac{1}{e^{h|\tau|}} \sim C N^{\alpha}(\log N)^{\beta}
$$

where $|\tau|$ is the cardinality of the finite orbit $\tau$ and $h$ denotes the topological entropy. For the usual orbit-counting function we find upper and lower bounds, together with numerical evidence to suggest that for actions of noncyclic groups there is no single asymptotic in terms of elementary functions.

\section{INTRODUCTION}

There is a well-understood analogy between the classical prime number theorem and Mertens' theorem on the one hand and closed orbits of dynamical systems on the other. For reasons that will become clear, we will think of an invertible map $T: X \rightarrow X$ as defining a $\mathbb{Z}$-action, also written $T$, by bijections of $X$. For any subgroup $L \leqslant \mathbb{Z}$, write

$$
\mathrm{F}_{T}(L)=\mid\left\{x \in X \mid T^{\ell}(x)=x \text { for all } \ell \in L\right\} \mid
$$

for the number of $L$-periodic points under the action, and $[L]$ for the index of $L$ in $\mathbb{Z}$. Write

$$
\mathrm{O}_{T}(L)=\frac{1}{[L]}\left|\left\{x \in X \mid T^{\ell}(x)=x \Leftrightarrow \ell \in L\right\}\right|
$$

for the number of $L$-orbits. Noting that $L$ is determined by the elements of an $L$-orbit, write $|\tau|=[L]$ if $\tau$ is an $L$-orbit, and call a set $\tau$ a closed orbit if it is an $L$-orbit for some $L$ of finite index.

Since $\mathrm{F}_{T}(L)=\sum_{L^{\prime} \geqslant L}\left[L^{\prime}\right] \mathrm{O}_{T}\left(L^{\prime}\right)$, Möbius inversion shows that

$$
\mathrm{O}_{T}(L)=\frac{1}{[L]} \sum_{L^{\prime} \geqslant L} \mu\left(\left|L^{\prime} / L\right|\right) \mathrm{F}_{T}\left(L^{\prime}\right) .
$$

Received by the editors June 25, 2007, and, in revised form, August 22, 2007, and May 5, 2008. 2000 Mathematics Subject Classification. Primary 22D40, 37A15, 37A35.

We thank Johannes Siemons and Shaun Stevens for their suggestions. This research was supported by E.P.S.R.C. grant EP/C015754/1.

(C)2008 American Mathematical Society Reverts to public domain 28 years from publication 
If $T$ is hyperbolic with respect to some metric structure on $X$ and if $T$ has topological entropy $h$, then the dynamical analogue of the prime number theorem takes the form

where

$$
\pi_{T}(N) \sim \frac{e^{(N+1) h}}{N}
$$

$$
\pi_{T}(N)=\mid\{\tau \mid \tau \text { is a closed orbit with }|\tau| \leqslant N\} \mid,
$$

and the dynamical analogue of Mertens' theorem is

$$
\mathrm{M}_{T}(N)=\log N+C+o(1)
$$

where

$$
\mathrm{M}_{T}(N)=\sum_{|\tau| \leqslant N} \frac{1}{e^{h|\tau|}}
$$

These are simplified statements of results due to Parry and Pollicott [1], Sharp 12] and others, motivated by results of Margulis [10] on orbits of geodesic flows.

Our purpose here is to explore possible extensions of these results to the setting of other group actions. For algebraic $\mathbb{Z}^{d}$-actions, the growth rate of periodic points has been studied by Lind, Schmidt, and Ward [7, Sec. 7], and some properties of a natural dynamical zeta function have been studied by Lind [8]. Despite this, the behaviour of closed orbits in these systems presents many difficulties: in particular, the growth rate of periodic points is only really understood for periods with good geometrical properties (corresponding to subgroups $L$ that are generated by vectors of length comparable to $[L]^{1 / d}$ ), and the dynamical zeta function is already a highly non-trivial object for the trivial action on a point. In order to find analogues of the prime number theorem and Mertens' theorem we need to take account of all orbits, no matter how awkwardly shaped. Because of the difficulties presented by this, and in order to expose some distinctive features of genuinely non-cyclic actions, we restrict our attention to full shifts, which are defined as follows. For any group $G$ and finite alphabet $B$, define the full $G$-shift $T: B^{G} \rightarrow B^{G}$ by

$$
\left(T_{g}(x)\right)_{h}=x_{g h} .
$$

It is clear that if $L \leqslant G$ is a subgroup of finite index, then

$$
\mathrm{F}_{T}(L)=b^{[L]},
$$

where $b=|B|$, and the topological entropy of the full shift is equal to $\log b$.

While our results are stated for this special kind of system, one of our aims is to highlight the way in which subgroup growth is inherently linked to orbit-growth properties. This is also true for single transformations but is much less noticeable because $\mathbb{Z}$ happens to have exactly one subgroup of each index.

\section{Closed orbits of Group actions}

Now consider an action $T$ of a finitely-generated group $G$ by bijections of a set $X$. Let $\mathcal{L}=\mathcal{L}(G)$ denote the set of subgroups of $G$ with finite index, and write $[L]$ for the index of $L$ in $G$. Define $\mathrm{F}_{T}(L)$ and $\mathrm{O}_{T}(L)$ using (11) and (2) for any $L \in \mathcal{L}$. In order to write down the analogue of (3), some terminology from combinatorics is needed. The partial order $\preccurlyeq$ defined by $L^{\prime} \preccurlyeq L$ if and only if $L \leqslant L^{\prime}$ makes $\mathcal{L}$ into a locally finite poset, which therefore has a Möbius function defined on intervals as follows (see Stanley [14, Sec. 3.7] for the details and examples; for convenience 
we use the relations $\leqslant, \geqslant$ rather than $\succcurlyeq, \preccurlyeq$ throughout). Define a function $\mu$ on pairs $\left(L^{\prime}, L\right) \in \mathcal{L}^{2}$ with $L \leqslant L^{\prime}$ by the properties

$$
\mu(L, L)=1 \text { for all } L \in \mathcal{L}
$$

and

$$
\mu\left(L^{\prime}, L\right)=-\sum_{L<L^{\prime \prime} \leqslant L^{\prime}} \mu\left(L^{\prime}, L^{\prime \prime}\right) \text { for all } L<L^{\prime} ;
$$

the symbol $\mu$ will always denote the Möbius function corresponding to $G$, whereas for a different group $H$ we write $\mu_{H}$. For the case $G=\mathbb{Z}$ dealt with in Section 1, the set $\mathcal{L}$ is in one-to-one correspondence with $\mathbb{N}$ via the correspondence $d \rightarrow d \mathbb{Z}$. Under this correspondence $d \mathbb{Z} \leqslant d^{\prime} \mathbb{Z}$ if and only if $d^{\prime} \mid d$, and $\mu\left(d^{\prime} \mathbb{Z}, d \mathbb{Z}\right)$ is just $\mu\left(d / d^{\prime}\right)$, the classical Möbius function. In order to understand one of the difficulties that arises in passing from $\mathbb{Z}$-actions to more general group actions, notice that one of the key estimates behind the results in $[3]$ is $|\mu| \leqslant 1$ on $\mathbb{Z}$, while on larger groups there is no reason to expect $\mu$ to be bounded.

For any group $G$, define the rank of $G$, written $r(G)$, to be the supremum over all finitely generated subgroups $H$ of the minimal cardinality of a generating set for $H$. The analogues of the prime number theorem and Mertens' theorem in our setting take the following form.

Theorem 1. Let $G$ be a finitely generated torsion-free nilpotent group, and let $T$ be the full $G$-shift on an alphabet with b symbols.

(1) There are positive constants $C_{1}$ and $C_{2}$ for which

$$
C_{1} N^{d(G)-2} \leqslant \frac{\pi_{T}(N)}{b^{N}} \leqslant C_{2} N^{r(G)-1},
$$

where $r(G)$ is the rank of $G$ and $d(G)$ is the rank of the abelianization of $G$. In the case $G=\mathbb{Z}^{d}, d \geqslant 2$,

$$
C_{1} N^{d-2} \leqslant \frac{\pi_{T}(N)}{b^{N}} \leqslant C_{2} N^{d-2}(\log N)^{d-1} .
$$

(2) There is a positive constant $C_{3}$, and there are non-negative constants $\alpha \in \mathbb{Q}$ and $\beta \in \mathbb{Z}$, with

$$
\mathrm{M}_{T}(N) \sim C_{3} N^{\alpha}(\log N)^{\beta},
$$

and in the case $G=\mathbb{Z}^{d}, d \geqslant 2$, there is a positive constant $C_{4}$ with

$$
\mathrm{M}_{T}(N) \sim C_{4} N^{d-1} .
$$

Proof. We begin by assembling some bounds for $a_{n}(G)$, the number of subgroups of $G$ with index $n$. By Grunewald, Segal and Smith [4, Prop. 1.1] (this is also shown in a different setting by Lind [8, Prop. 4.2]) we have

$$
a_{n}\left(\mathbb{Z}^{d}\right)=\sum_{k \mid n} a_{n / k}\left(\mathbb{Z}^{d-1}\right) k^{d-1},
$$

so, in particular,

$$
a_{n}\left(\mathbb{Z}^{d}\right) \geqslant n^{d-1} \text { for all } n, d \geqslant 1 .
$$

It follows that

$$
a_{n}(G) \geqslant n^{d(G)-1} .
$$


From [9, Lemma 1.4.1],

$$
a_{n}(G)<n^{r(G)} \text { for } n>1,
$$

so $s_{n}(G)=\sum_{k=1}^{n} a_{k}(G)<n^{r(g)+1}$.

Notice that

$$
\begin{aligned}
\pi_{T}(N)=\sum_{|\tau| \leqslant N} 1 & =\sum_{[L] \leqslant N} \mathrm{O}_{T}(L) \\
& =\sum_{[L] \leqslant N} \frac{1}{[L]} \sum_{L^{\prime} \geqslant L} \mathrm{~F}_{T}\left(L^{\prime}\right) \mu\left(L^{\prime}, L\right) \\
& =\sum_{[L] \leqslant N} \frac{1}{[L]} \mathrm{F}_{T}(L)+\underbrace{\sum_{[L] \leqslant N} \frac{1}{[L]} \sum_{L^{\prime}>L} \mathrm{~F}_{T}\left(L^{\prime}\right) \mu\left(L^{\prime}, L\right)}_{\Sigma_{N}} .
\end{aligned}
$$

$$
F_{T}\left(L^{\prime}\right) \leqslant b^{[L] / 2} \text { for all } L<L^{\prime} .
$$

Lemma 2. There exists a constant $C_{5}$ such that $\left|\mu\left(L^{\prime}, L\right)\right| \leqslant e^{C_{5}(\log [L])^{2}}$ for $L \leqslant L^{\prime}$.

Proof. If $L$ is not normal in $L^{\prime}$, then (since passing to the normal closure is a closure operator on $\mathcal{L}$ ), Crapo's Theorem (see [1, Thm. 1]) shows that $\mu\left(L^{\prime}, L\right)=0$.

If $L$ is normal in $L^{\prime}$, then $\mu\left(L^{\prime}, L\right)=\mu_{L^{\prime} / L}\left(L^{\prime} / L,\{0\}\right)$, and by Kratzer and Thévenaz [5, Prop. 2.4], $\mu_{L^{\prime} / L}\left(L^{\prime} / L,\{0\}\right)=0$ if $L^{\prime} / L$ is not a product of elementary abelian groups and is bounded by

$$
\prod_{i=1}^{r} p_{i}^{n_{i}\left(n_{i}-1\right) / 2}
$$

if $L^{\prime} / L \cong \prod_{i=1}^{r}\left(\mathbb{Z} / p_{i} \mathbb{Z}\right)^{n_{i}}$. In this case, writing $\left|L^{\prime} / L\right|=k$ and $v_{i}(\cdot)=\operatorname{ord}_{p_{i}}(\cdot)$, it follows that

$$
\left(\mu_{L^{\prime} / L}\left(L^{\prime} / L,\{0\}\right)\right)^{2} \leqslant \prod_{i=1}^{r} p_{i}^{v_{i}(k)^{2}}=\prod_{i=1}^{r}\left(p_{i}^{v_{i}(k)}\right)^{v_{i}(k)} \leqslant\left(\prod_{i=1}^{r} p_{i}^{v_{i}(k)}\right)^{\log _{2} k}=k^{\log _{2}(k)},
$$

so there is a constant $C_{5}$ with

$$
\left|\mu_{L^{\prime} / L}\left(L^{\prime} / L,\{0\}\right)\right| \leqslant e^{C_{5}(\log [L])^{2}} .
$$

Therefore, (10) gives

$$
\begin{aligned}
\frac{\Sigma_{N}}{b^{N}} & \leqslant \frac{1}{b^{N}} \sum_{[L] \leqslant N} \frac{1}{[L]} b^{[L] / 2} e^{C_{5}(\log [L])^{2}} s_{[L]}(G) \\
& \leqslant \frac{1}{b^{N}} \sum_{n=1}^{N} \frac{1}{n} b^{n / 2} e^{C_{5}(\log n)^{2}} a_{n}(G) s_{n}(G) \\
& \leqslant \frac{1}{b^{N}} \sum_{n=1}^{N} \frac{1}{n} b^{n / 2} e^{C_{5}(\log n)^{2}} n^{2 r(G)+1} \quad \text { by (9) } \\
& \leqslant b^{-N / 2} N^{2 r(G)+2} e^{C_{5}(\log N)^{2}} \rightarrow 0 \text { as } N \rightarrow \infty .
\end{aligned}
$$


It follows that

$$
\frac{\pi_{T}(N)}{b^{N}}+o(1)=\frac{1}{b^{N}} \sum_{[L] \leqslant N} \frac{1}{[L]} F_{T}(L)=\frac{1}{b^{N}} \sum_{n=1}^{N} \frac{a_{n}(G)}{n} b^{n} .
$$

By partial summation,

$$
\sum_{n=1}^{N} n^{e} b^{n}=\frac{b}{b-1} N^{e} b^{N}+O\left(N^{e-1} b^{N}\right)
$$

for any $e \geqslant 1$ since $b \geqslant 2$. Thus by (8) and (9), part (1) of the theorem in the nilpotent case follows from (11).

Now consider the case $G=\mathbb{Z}^{d}$. The estimate (7) in place of (8) gives the stated lower bound by the same argument. For the upper bound we use the following lemma.

Lemma 3. For $n \geqslant 2$,

$$
a_{n}\left(\mathbb{Z}^{d}\right) \leqslant 3^{d} n^{d-1}(\log n)^{d-1} .
$$

Proof. If $d=2$, then (6) gives $a_{n}\left(\mathbb{Z}^{2}\right)=\sigma(n)=\sum_{d \mid n} d$, and a simple argument shows that $\sigma(n) \leqslant 3 n \log (n)$ for $n \geqslant 2$. Now assume the statement of the lemma, and notice that by (6) we have

$$
\begin{aligned}
a_{n}\left(\mathbb{Z}^{d+1}\right) & =\sum_{k \mid n} a_{n / k}\left(\mathbb{Z}^{d}\right) k^{d} \\
& \leqslant 3^{d} \sum_{k \mid n}(n / k)^{d-1}(\log (n / k))^{d-1} k^{d} \quad \text { by hypothesis } \\
& \leqslant 3^{d} n^{d-1}(\log n)^{d-1} \sum_{k \mid n} k \\
& \leqslant 3^{d+1} n^{d}(\log n)^{d} \text { for } n \geqslant 2 .
\end{aligned}
$$

Now there are positive constants $C_{6}$ and $C_{7}$ with

$$
\begin{aligned}
\frac{1}{b^{N}} \sum_{n=1}^{N} \frac{a_{n}\left(\mathbb{Z}^{d}\right)}{n} b^{n} & \leqslant C_{6} \frac{1}{b^{N}} \sum_{n=1}^{N} n^{d-2}(\log n)^{d-1} b^{n} \quad \text { by Lemma } 3 \\
& \leqslant C_{6} \frac{1}{b^{N}}(\log N)^{d-1} \sum_{n=1}^{N} n^{d-2} b^{n} \\
& \leqslant C_{7} N^{d-2}(\log N)^{d-1} \text { by (12), }
\end{aligned}
$$

giving the upper bound by (11). 
Turning to the analogue of Mertens' theorem, notice that

$$
\begin{aligned}
\mathrm{M}_{T}(N)=\sum_{|\tau| \leqslant N} \frac{1}{b^{|\tau|}} & =\sum_{n=1}^{N} \frac{1}{b^{n}} \sum_{[L]=n} \mathrm{O}_{T}(L) \\
& =\sum_{n=1}^{N} \frac{1}{b^{n}} \sum_{[L]=n} \frac{1}{n} \sum_{L^{\prime} \geqslant L} \mu\left(L^{\prime}, L\right) \mathrm{F}_{T}\left(L^{\prime}\right) \\
& =\sum_{n=1}^{N} \frac{a_{n}(G)}{n}+\underbrace{\sum_{n=1}^{N} \frac{1}{n b^{n}} \sum_{[L]=n} \sum_{L^{\prime}>L} \mu\left(L^{\prime}, L\right) b^{\left[L^{\prime}\right]}}_{\Delta_{N}},
\end{aligned}
$$

and by (9) and Lemma 2 we have

$$
\left|\Delta_{N}\right| \leqslant \sum_{n=1}^{N} \frac{1}{n b^{n}} n^{r(G)+1} b^{n / 2} e^{C_{5}(\log (n / 2))^{2}}=O(1) .
$$

It follows that

$$
\mathrm{M}_{T}(N)=\sum_{n=1}^{N} \frac{a_{n}(G)}{n}+O(1)
$$

Now by a deep theorem of du Sautoy and Grunewald [2, Thm. 1.1], there is a constant $C_{8}$ and there are non-negative constants $\gamma \in \mathbb{Q}, \delta \in \mathbb{Z}$ with

$$
s_{n}(G) \sim C_{8} n^{\gamma}(\log n)^{\delta} .
$$

If $\gamma \geqslant 1$, then it follows by partial summation that $\mathrm{M}_{T}(N)$ has the asymptotic stated in part (2) with $\alpha=\gamma-1$ and $\beta=\delta$. If $\gamma<1$, then partial summation shows that $\mathrm{M}_{T}(N)$ is bounded, so we may take $\alpha=\beta=0$. In the case $G=\mathbb{Z}^{d}$, the well-known relation

$$
\sum_{n=1}^{\infty} \frac{a_{n}\left(\mathbb{Z}^{d}\right)}{n^{z}}=\zeta(z) \zeta(z-1) \cdots \zeta(s-d+1)
$$

gives, via a Tauberian theorem (see [2]), an asymptotic of the form

$$
s_{n}\left(\mathbb{Z}^{d}\right) \sim C_{9} n^{d}
$$

which gives the conclusion.

\section{EXAmples}

There are two clear directions in which Theorem 1 is limited. It applies to a very special class of dynamical systems, and Examples 3 and 4 indicate some of the obstacles to extending it to a broader class of systems. Moreover, the estimates used are naïve and no doubt more sophisticated techniques could improve the bounds.

Example 1. It is not surprising that $\mathrm{M}_{T}$ is less sensitive to volatility in $\mathrm{O}_{T}(L)$ than is $\pi_{T}$; in this example we explain something about the extent of this difference in the simplest non-cyclic case $G=\mathbb{Z}^{2}$ with $b=2$. By Theorem 1, $\mathrm{M}_{T}(N) \sim C_{3} N$. On the other hand, by (6) we have $a_{n}\left(\mathbb{Z}^{2}\right)=\sigma(n)$, so from (11) we have

$$
\frac{\pi_{T}(N)}{2^{N}}=\frac{1}{2^{N}} \sum_{n=1}^{N} \frac{\sigma(n)}{n} 2^{n}+o(1) .
$$


The graph in Figure 1 illustrates the asymptotic in (13) and the erratic behaviour of $\pi_{T}(N)$ as a function of $N$.

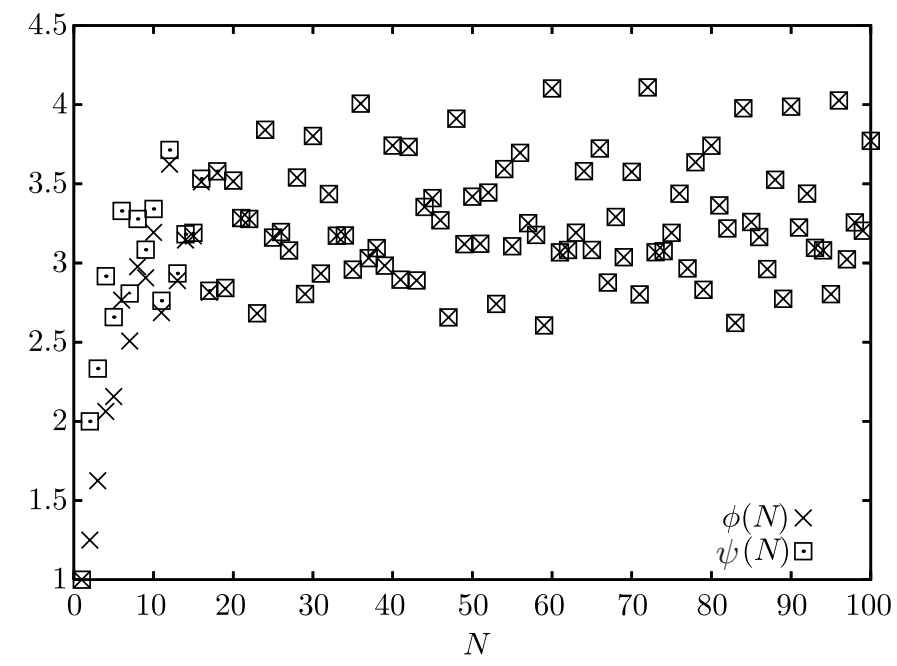

Figure 1. Plot of $\phi(N)=\frac{\pi_{T}(N)}{2^{N}}$ and $\psi(N)=\frac{1}{2^{N}} \sum_{n=1}^{N} \frac{\sigma(n)}{n} 2^{n}$.

Example 2. A simple example with $\beta>0$ in Theorem 1(2) is given by the discrete Heisenberg group

$$
G=\left\{\left(\begin{array}{lll}
1 & x & y \\
0 & 1 & z \\
0 & 0 & 1
\end{array}\right) \mid x, y, z \in \mathbb{Z}\right\},
$$

which has

$$
s_{n}(G) \sim \frac{\zeta(2)^{2}}{2 \zeta(3)} N^{2} \log N
$$

by Smith [13] (see also [2, Sect. 1]). It follows that

$$
\begin{aligned}
\mathrm{M}_{T}(N) & \sim \frac{s_{N}(G)}{N}+\int_{1}^{N} \log t \mathrm{~d} t \\
& \sim\left(\frac{\zeta(2)^{2}}{2 \zeta(3)}+1\right) N \log N
\end{aligned}
$$

The bounds for $\pi_{T}(N) / b^{N}$ are of course much weaker,

$$
0<C_{1} \leqslant \frac{\pi_{T}(N)}{b^{N}} \leqslant C_{2} N^{2} .
$$

A natural setting to seek results of this sort would be expansive algebraic $\mathbb{Z}^{d_{-}}$ actions, because their growth rate of periodic points is available from [7]. However, this growth rate result concerns a sequence of subgroups $L_{n}$ with the property that $\mathrm{d}\left(0, L_{n} \backslash\{0\}\right) \rightarrow \infty$ as $n \rightarrow \infty$ (for the Euclidean metric $\mathrm{d}$ on $\mathbb{Z}^{d}$ ). As mentioned in the introduction, genuine orbit-counting results should include all orbits. The examples above describe some of the implications of erratic behaviour in the map $n \mapsto a_{n}(G)$; the next two examples concern two different ways in which 
the map $\mathrm{F}: L \mapsto \mathrm{F}_{T}(L)$ may be erratic, in contrast to the full shift where $\mathrm{F}_{T}(L)$ is a regularly growing function of the index $[L]$ alone.

Example 3. For expansive $\mathbb{Z}^{d}$-actions without any entropy assumption, $F$ may be sensitive to small changes in subgroups of fixed shape. For example, in Ledrappier's $\mathbb{Z}^{2}$-action (see [6]) we have

$$
\mathrm{F}_{T}\left(\left(2^{k}, 0\right) \mathbb{Z} \oplus\left(0,2^{k}\right) \mathbb{Z}\right)=1
$$

for all $k \geqslant 1$ and

$$
\mathrm{F}_{T}\left(\left(2^{k}-1,0\right) \mathbb{Z} \oplus\left(0,2^{k}-1\right) \mathbb{Z}\right) \rightarrow \infty
$$

as $k \rightarrow \infty$ (see [15, Ex. 3.3]). Notice that the quantity $b$ appearing in Theorem 1 is $e^{h}$, where $h$ denotes the topological entropy of the action. For this example, $h(T)=0$ and the expression $\mathrm{M}_{T}(N)$ collapses to $\pi_{T}(N)$, and so no averaging is taking place.

Example 4. Expansive $\mathbb{Z}^{d}$ actions with completely positive entropy do have the property that $\mathrm{F}_{T}(L)$ is not very sensitive to changes in $[L]$ for a fixed shape of a subgroup. However, they may still be sensitive to the shape of $L$ for a fixed index $[L]$. For example, the $\mathbb{Z}^{2}$-action on $X^{\mathbb{Z}}$, where $X$ is the Pontryagin dual of $\mathbb{Z}\left[\frac{1}{2}\right]$, defined by $\left(T_{(1,0)}(x)\right)_{k}=2 x_{k}(\bmod 1)$ and $\left(T_{(0,1)}(x)\right)_{k}=x_{k+1}$ has

$$
\mathrm{F}_{T}((n, 0) \mathbb{Z} \oplus(0,1) \mathbb{Z})=2^{n}-1,
$$

while

for all $n \geqslant 1$.

$$
\mathrm{F}_{T}((1,0) \mathbb{Z} \oplus(0, n) \mathbb{Z})=1
$$

\section{REFERENCES}

[1] H. H. Crapo, Möbius inversion in lattices, Arch. Math. (Basel) 19 (1968), 595-607 (1969). MR0245483(39:6791)

[2] M. du Sautoy and F. Grunewald, Analytic properties of zeta functions and subgroup growth, Ann. of Math. (2) 152 (2000), no. 3, 793-833. MR.1815702 (2002h:11084)

[3] G. Everest, R. Miles, S. Stevens, and T. Ward, Orbit-counting in non-hyperbolic dynamical systems, J. Reine Angew. Math. 608 (2007), 155-182. MR2339472

[4] F. J. Grunewald, D. Segal, and G. C. Smith, Subgroups of finite index in nilpotent groups, Invent. Math. 93 (1988), no. 1, 185-223. MR943928 (89m:11084)

[5] C. Kratzer and J. Thévenaz, Fonction de Möbius d'un groupe fini et anneau de Burnside, Comment. Math. Helv. 59 (1984), no. 3, 425-438. MR761806 (86k:20011)

[6] F. Ledrappier, Un champ markovien peut être d'entropie nulle et mélangeant, C. R. Acad. Sci. Paris Sér. A-B 287 (1978), no. 7, A561-A563. MR512106 (80b:28030)

[7] D. Lind, K. Schmidt, and T. Ward, Mahler measure and entropy for commuting automorphisms of compact groups, Invent. Math. 101 (1990), no. 3, 593-629. MR1062797 (92j:22013)

[8] D. A. Lind, A zeta function for $\mathbb{Z}^{d}$-actions, Ergodic theory of $\mathbb{Z}^{d}$ actions (Warwick, 19931994), London Math. Soc. Lecture Note Ser., vol. 228, Cambridge Univ. Press, Cambridge, 1996, pp. 433-450. MR.1411232 (97e:58185)

[9] Alexander Lubotzky and Dan Segal, Subgroup growth, Progress in Mathematics, vol. 212, Birkhäuser Verlag, Basel, 2003. MR1978431 (2004k:20055)

[10] G. A. Margulis, On some aspects of the theory of Anosov systems, Springer Monographs in Mathematics, Springer-Verlag, Berlin, 2004, with a survey by Richard Sharp: Periodic orbits of hyperbolic flows; translated from the Russian by Valentina Vladimirovna Szulikowska. MR2035655 (2004m:37049)

[11] W. Parry and M. Pollicott, An analogue of the prime number theorem for closed orbits of Axiom A flows, Ann. of Math. (2) 118 (1983), no. 3, 573-591. MR727704 (85i:58105)

[12] R. Sharp, An analogue of Mertens' theorem for closed orbits of Axiom A flows, Bol. Soc. Brasil. Mat. (N.S.) 21 (1991), no. 2, 205-229. MR 1139566 (93a:58142) 
[13] G. C. Smith, Zeta functions of torsion free finitely generated nilpotent groups, Ph.D. thesis, Manchester (UMIST), 1983.

[14] R. P. Stanley, Enumerative combinatorics. Vol. 1, Cambridge Studies in Advanced Mathematics, vol. 49, Cambridge University Press, Cambridge, 1997, with a foreword by Gian-Carlo Rota; corrected reprint of the 1986 original. MR.1442260 (98a:05001)

[15] T. Ward, Periodic points for expansive actions of $\mathbb{Z}^{d}$ on compact abelian groups, Bull. London Math. Soc. 24 (1992), no. 4, 317-324. MR1165372 (93h:22010)

School of Mathematics, KTH, SE-100 44, Stockholm, Sweden

School of Mathematics, University of East Anglia, Norwich, NR4 7TJ, United KingDOM 